\title{
Morning lectures
}




\title{
Hypercoagulability and thrombosis in sickle cell anemia and thalassemia
}

\author{
David Varon, Eliezer A. Rachmilewitz
}

Coagulation Unit, Hadassah-Hebrew University Medical Center, Jerusalem, Israel

Sickle cell disease (SCD) and thalassemia are hereditary hemolytic anemias resulting from either a point mutation in the $\beta$-globin chain, and partial or complete deficiency of either $\alpha$ or $\beta$ chains of hemoglobin A respectively.

The increased life expectancy of these patients due to improved therapy resulted in the development of complications which were not recognized earlier. These complications include an increased rate of deep venous thrombosis (DVT), pulmonary embolism (PE), as well as arterial thrombosis, stroke, and a high incidence of asymptomatic ischemic brain damage.

Although the pathophysiology of hypercoagulable state in the two hemoglobinopathies might not be identical, red blood cell (RBC) abnormalities, platelet activation, endothelial damage, and other systemic factors were implicated in both diseases. For instance, an increased level of negatively charged phospholipids (phosphatidylserine), which is expressed on the outer layer of RBC, platelets and maybe in other cell membranes within the vascular compartment, was found in both diseases. The abnormal distribution of membrane phospholipids is probably driven by oxidative stress due to the generation of free oxygen radicals which are catalyzed by free iron species (labile iron pool) in the RBC, partially derived from the plasma (labile plasma iron). This common denominator explains the findings of increased RBC and platelet reactivity associated with increased thrombin generation, resulting in a hypercoagulable state. An increased rate of thromboembolism was found mainly in patients who underwent splenectomy, most probably due to the high number of pathological $\mathrm{RBC}$ and platelets which are cleared by the spleen. Consequently, these findings may promote the development of innovative approaches to prevent thromboembolic complications in SCD and thalassemia. 
Pathophysiology of Haemostasis and Thrombosis

\title{
Inflammation and atherothrombosis
}

\author{
Erik Stroes
}

F4.211, Dept of Vascular Medicine, AMC, Amsterdam, the Netherlands

Atherosclerosis as well as the subsequent progression towards cardiovascular events are considered to, at least partially, be a consequence of chronic inflammatory activity. In recent years, the relation between lipids, inflammation, atherosclerosis and thrombosis is increasingly being acknowledged.

Consequences for diagnosis and therapy of inflammatory patients will be discussed. 


\title{
Leukocytosis as a thrombosis risk factor
}

\author{
Tiziano Barbui
}

Department of Hematology , Ospedali Riuniti, Bergamo, Italy

\begin{abstract}
Arterial and venous thrombosis are multifactorial diseases and are associated with multiple interacting genetic and environmental risk factors. For arterial disease, some of these risk factors can be "atherogenic" promoting progression of occlusive atherosclerosis (eg, smoking, hypertension, cholesterol, diabetes), whereas others may be "thrombogenic" (eg, obesity, diabetes, immobility, estrogens, infections, smoking) promoting rupture of atheromatous plaques and superadded thrombosis. There is now increasing evidence that arterial disease may directly promote venous thromboembolic events and that activation of inflammation and hemostatic system plays a role in progression of atherosclerosis. In this context, laboratory and epidemiologic investigations have attributed a major pathogenetic role to leukocytes (WBC) both in terms of promoting atherogenesis and thrombogenesis. Thus, it is not surprising that a longterm exposition of activated leukocytes, as shown in myeloproliferative neoplasms (MPNs) such as Polycythemia Vera (PV), Essential Thrombocythemia (ET) and Primary Myelofibrosis (PMF), can damage vascular endothelium and promote thrombosis. Mechanisms of this action involve multiple interactions with platelets and vessel walls as shown by many laboratory studies. The natural history of these diseases is marked by an high rate of cardiovascular complications that are major cause of morbidity and mortality as shown in prospective and in several retrospective studies.
\end{abstract}

Correspondence: Department of Hematology, Ospedali Riuniti, Largo Barozzi 1, Bergamo,Italy.tbarbui@ospedaliriuniti.bergamo.it
The largest and most recent prospective study evaluating risk factors for survival and thrombosis in PV is the European Collaboration on Low-dose Aspirin in Polycythemia (ECLAP). In this study, the incidence of cardiovascular complications was higher in patients aged more than 65 years $(5.0 \%$ patient-year, hazard ratio 2.0 , $95 \%$ confidence interval $[\mathrm{CI}] 1.22-3.29, P<0.006)$ or with a history of thrombosis $(4.93 \%$ patient-year, hazard ratio $1.96,95 \% \mathrm{CI} 1.29-2.97, P=0.0017)$ than in younger subjects with no history of thrombosis $(2.5 \%$ patientyear, reference category). Patients with both a history of thrombosis and age more than 65 years had the highest risk of cardiovascular events during follow-up (10.9\% patientyear, hazard ratio $4.35,95 \%$ CI $2.95-6.41, P<0.0001)$. Other significant predictors of survival and cardiovascular morbidity were smoking, diabetes mellitus, and congestive heart failure.

In a more recent re-evaluation of the predictors of thrombosis, a relevant role for the white blood cell count has been found. In baseline and time-dependent multivariable analysis, patients with a WBC count above $15 \times 109 / \mathrm{L}$, compared with those with a WBC count below 10x109/L had a significant increase in the risk of myocardial infarction (HR 1.71; 95\% confidence interval [CI] 1.10-2.65).

In a case-control analysis, the highest leukocyte percentile compared with the lowest one, showed increase mortality, more frequent major thrombosis and evolution 
to leukemia. In contrast, this study failed to show any association between platelet count and thrombotic events and in multivariable analysis could not demonstrate an independent role of hematocrit levels in the range of 40 to $52 \%$. To evaluate whether an increased leukocyte count was associated with thrombosis also in Essential Thrombocythemia (ET) the clinical course of 439 patients was analysed. The strength of the association was measured at diagnosis or before thrombotic events by multivariable analyses carried out using data at baseline as well as time-varying covariates. The results showed that: (1) an increased leukocyte count at diagnosis was associated with thrombosis during follow-up ("baseline analysis," relative risk [RR] 2.3, 95\% confidence interval [CI] 1.4-3.9, $P=.001$ ); (2) hydroxyurea (HU) lowered leukocytosis and reduced the strength of the association between leukocytosis and thrombosis ("time-dependent analysis," RR 1.6, 95\% CI 0.9-2.0, not significant [NS]); (3) the association of leukocytosis and thrombosis was more evident in untreated low-risk patients (RR 2.7, 95\% CI 1.2-6.4, $P=.01)$ compared with HU-treated highrisk patients (RR 1.6, 95\% CI $0.8-3.2, \mathrm{NS}$ ); and (4) the presence of JAK2 V617F was not identified as a risk factor for thrombosis during follow-up despite a significant association between the mutation and leukocytosis.

There are now laboratory data to explain the thrombogenic potential of leukocytosis. We found an increase of in vivo platelet-leukocyte interaction, particularly in ET patients carrying the V617F JAK2 mutation; this was associated with activation of the plasmatic hemostatic system thus causing an hypercoagulable status. However, in spite of these findings documenting a role of WBC as risk factor for events in PV and ET, there are some limitations to include "leukocytosis" in the algorithms to stratify patients to different treatments. First, it is unknown whether leukocytosis per se has an incremental role in addition to conventional risk factors to discriminate the group that will have thrombosis and the group that will not. Second, the best cut-off value of leukocytosis, with the highest sensitivity and specificity, that will discriminate the groups with different probability of events has to be assessed. Third, this link deserves further analysis and validation in a larger series of patients to provide a solid basis for this test to be prognostic of thrombosis in individual patients. 


\title{
Familial thrombophilia without a positive laboratory screening
}

\author{
Jacqueline Conard
}

Dept of Biological Hematology, Hotel-Dieu Hospital, Paris, France

\begin{abstract}
Thrombophilia is defined as a predisposition to venous thromboembolism (VTE). It may be related to an acquired or hereditary biological change. Antiphospholipid syndrome is the main acquired thrombophilia but in exceptional cases, it is familial. Main hereditary thrombophilias are: deficiencies in antithrombin, protein $\mathrm{C}$, protein $\mathrm{S}$ and mutations of Factor V Leiden and Factor II G20210A: they are found in $40-60 \%$ of patients with VTE history. Other biological thrombophilias have been found: hyperhomocysteinemia, increased levels of factor VIII, IX, XI, molecular changes in thrombomodulin, factor XII, endothelial protein C receptor, tissue factor pathway inhibitor, plasminogen activator inhibitor (PAI). Odds ratios appear to be lower and lower and the frequency and relevance of the latter changes in familial thrombophilia are not really known since studies did not generally include all these tests in a same population of patients. However, in some families, severe VTE events are
\end{abstract}

reported and still unexplained. Other presently unknown biological thrombophilias are likely but they will probably be rare and/or associated with low risks. Individual and environmental risks factors are frequently present in patients with familial thrombophilia: contraception, pregnancy, surgery, immobilization, treatments for cancer, atypical antipsychotics. Some of them may be familial (obesity), others may be related to pollution, as recently reported. A family might be exposed to a same risk factor of thrombosis without meaning a hereditary thrombophilia. In conclusion, familial thrombophilia exists. Some wellknown or less well-known biological thrombophilias are found, some are still unknown. But associated individual and environmental risk factors should be taken into account more and better evaluated. A score would be useful to determine the individual risk of a patient with a personal and/or familial history of VTE. 


\title{
Haemotransfusion in coronary heart disease: friend or foe?
}

\author{
Maddalena Lettino
}

Fondazione IRCCS Policlinico S.Matteo Hospital, Pavia, Italy

The problems concerning haemotransfusions in coronary artery disease have been raised in the setting of acute coronary syndromes (ACS). New antithrombotic therapy associations have been introduced in the last decade in order to prevent thrombotic complications in the early phase of unstable angina, NSTEMI and STEMI in patients at high risk of cardiovascular death, myocardial infarction (MI) or stroke. Moreover, advances in percutaneous coronary interventions (PCI) have led to more widespread use of these procedures in the same set of patients, with a consequent increase in the risk of bleeding, particularly in elderly subjects, in women or in patients with several comorbidities like chronic renal failure. Recent studies, however, have identified major bleeding complications in ACS as an important predictor of increased mortality. In this setting it has been reported a potential adverse role of blood transfusions, specifically in patients treated with
PCI during the acute phase. Several potential mechanisms linking the delivery of stored red blood cells by transfusions with excess mortality are: a reduced tissue extraction of oxygen, a reduced nitric oxide transportation, a premature clearance of transfused red blood cells by the spleen or tissue ischemia caused by the plugging of blood cells in the microvasculature, due to hemorheological alterations of them, like shape change, increase aggregability and decrease deformability.

It is important to emphasize, however, that none of the published studies have established a causal connection between blood transfusion and increased mortality after PCI. Important questions regarding appropriate transfusion thresholds and the optimal storage conditions of red blood cells remain still unanswered and need to be addressed by future adequately powered studies. 


\title{
New antiplatelet agents: why they are badly needed by the cardiologist
}

\author{
Piera Merlini
}

Divisione di Cardiologia, Ospedale Niguarda, Milan, Italy

Each year, approximately 750,000 patients are admitted to hospitals in the United States with acute coronary syndromes (ACS), defined as unstable angina (UA) or myocardial infarction (MI) with or without ST-segment elevation (STE). Patients with moderate- to high-risk ACS are optimally managed using an early invasive approach involving angiography followed by percutaneous coronary intervention (PCI) or coronary artery bypass grafting (CABG). Acute coronary syndromes and coronary interventions carry an inherent risk of thrombotic complications.

Therefore, patients must receive periprocedural adjunctive pharmacotherapy targeted at inhibition of platelet aggregation and the coagulation cascade. However, the very mechanisms that confer the benefits of these agents also increase risk of bleeding. Thus, selection of appropriate pharmacotherapy requires close attention to the delicate balance between reducing the risk of ischemic events and minimizing bleeding risk.

A broad range of antiplatelet agents is available,including aspirin, thienopyridines, and glycoprotein IIb-IIIa inhibitors, all of which have shown considerable efficacy and excellent safety.

Selecting the appropriate antiplatelet therapy requires close attention to the delicate balance between reducing risk of ischemic events while minimizing bleeding risk. The broad range of available agents, while permitting to tailor pharmacotherapy to individual patients, also complicates the selection of optimal regimens. Platelet physiology provides an underpinning for the rationale behind pharmacotherapeutic strategies for patients with non-ST segment elevated acute coronary syndromes (NSTE ACS) undergoing percutaneous coronary intervention (PCI). The same mechanisms of action that confer anti-ischemic benefit with antiplatelet agents may also be associated with increased risk. In the context of ACS and PCI, antiplatelet agents are used in complex strategies and combinations with other pharmacotherapies targeted at alleviating ischemic symptoms and reducing ischemic risk. Accounting for individual patient risk factors, timing of treatment, and dosage of antiplatelet medications minimizes risk while optimizing outcomes. This review will results from clinical trials of thienopyridines (clopidogrel, ticlopidine, and the newer prasugrel), the new P2Y12 antagonists ticagrelor and cangrelor, glycoprotein IIb-IIIa inhibitors (abciximab, eptifibatide, tirofiban), and the direct thrombin inhibitor bivalirudin. Recommendations include clopidogrel for upstream use if discontinued 5 days before coronary angiographic bypass graft. Bivalirudin remains a reasonable treatment choice in patients at low to moderate risk; and glycoprotein IIb-IIIa inhibitors confer anti-ischemic benefit with little incremental bleeding risk when individual patient factors are taken into account for their dosing. Increased awareness of the factors contributing to risks and benefits associated with the available antiplatelet agents will help guide physicians in choosing optimal regimens for all patients. 


\title{
Is aspirin less effective in patients with diabetes?
}

\author{
Sergio Coccheri
}

University of Bologna, Bologna, Italy

\begin{abstract}
Although aspirin remains the "reference" and "first line" antiplatelet agent, several limitations emerged in recent years. Concepts as relative vs absolute risk reduction, residual vascular risk and resistance to aspirin or clopidogrel are presently items of wide debate.

Patients with Type 2 diabetes carry a high risk of cardiovascular events and would therefore appear ideal candidates to aspirin therapy. However, results of relevant clinical trials are deceiving. Distinction between 'primary' and 'secondary' prevention is less clear in patients with diabetes, a condition that is a potent risk factor per se, often associated with additional factors as hypertension, dyslipidemia, obesity and others. Diabetics are largely inhomogeneous in relation to this cluster and their global risk level is highly variable. Already in the antiplatelet meta-analysis of 2002 the risk reduction in diabetics was low and non-significant, especially under aspirin. In the Primary Prevention Study, aspirin was totally ineffective in
\end{abstract}

the diabetic group. In the JPAD primary prevention study, the results were inconclusive. More recently, in diabetics with only asymptomatic PAD (POPADAD Study) aspirin again failed to prevent cardiovascular events. In a final meta-analysis (2009) aspirin once more resulted little effective except for a reduction of non-fatal AMI, similarly to primary prevention in non-diabetics.

Thus, aspirin appears scarcely effective in patients without previous cardiovascular events in diabetics as in non-diabetics, and may also be less effective in those with events in presence of diabetes. A high prevalence of biological aspirin resistance was found in diabetic patients, probably due to: glycation of COX1, fast platelet turnover, activation of alternative mechanisms of TxA2 production. As clopidogrel is also subject to resistance in many clinical settings, new drugs as prasugrel, or different mechanisms of action (e.g. picotamide) are among the candidate drugs for antiplatelet prevention in diabetes. 


\title{
Which antithrombotic and antiplatelet therapies in the ambulance for acute coronary syndrome?
}

\author{
Marco Tubaro
}

ICCU, Cardiovascular Department, San Filippo Neri Hospital, Rome, Italy

\begin{abstract}
The more common pathophysiologic mechanism of acute coronary syndromes (ACS) is the rupture of the fibrous cap of an unstable coronary atherosclerotic plaque, which elicits a rapid local thrombosis with partial or complete occlusion of the coronary artery. Therefore, antithrombotic drugs have a pivotal role in ACS treatment and the efficacy of this pharmacologic therapy is inversely related with the time delay between the onset of symptoms and drug delivery.

With the aim to reduce this delay at most, pre-hospital administration of antithrombotic drugs is strongly advisable, particularly by Emergency Medical Services personnel on the ambulance. Because the coronary thrombus is the result of coagulation and platelet activation, and of fibrin production, three classes of drugs are of interest for the ambulance staff: fibrinolytic, antithrombotic and antiplatelet agents.

Fibrinolytic therapy is more effective if administered in the pre-hospital setting than in the in-hospital phase, particularly within the first 'golden hour' after the onset of symptoms. Recent data have shown that pre-hospital fibrinolysis by ambulance staff, followed by trasportation to a PCI-capable centre, yields survival results comparable
\end{abstract}

to those of primary PCI.

Unfractionated heparin (UFH) improves infarct-related artery patency when given with rtPA, while enoxaparin showed an improved net clinical benefit in comparison with UFH. No data are available on the pre-hospital use of the newer antithrombotics, like fondaparinux and bivalirudin.

Aspirin has been used in ACS for many years, given as soon as possible in oral non enteric-coated formulations; more recently, double antiplatelet therapy, with clopidogrel added to aspirin, demonstrated to further improve patients' outcome, both in case of thrombolytic therapy and primary PCI. Because of a lag-phase of at least 6 hours between clopidogrel administration and effectiveness, this drug has to be given as soon as possible, together with aspirin, in the ambulance.

Prasugrel is a new and more effective thienopyridine derivative, which improves STEMI patients' clinical outcome, in comparison with clopidogrel. However, we do not have data on the use of this drug in the ambulance. The pre-hospital use of Gp IIb/IIIa inhibitors before primary PCI does not offer clear advantages when compared with the administration in cath lab. 


\title{
Biomarkers for the diagnosis of venous thromboembolism
}

\author{
Arnaud Perrier
}

Division of General Internal Medicine, Geneva University Hospitals, Geneva, Switzerland

\begin{abstract}
Among the various biomarkers that have been evaluated in the context of venous thromboembolism (VTE), only D-dimer has proved useful for diagnosis, mainly for ruling out VTE when negative. Natriuretic peptides (BNP or NTproBNP) and troponin are restricted to risk stratification in patients with diagnosed pulmonary embolism (PE). Nevertheless, there are numerous D-dimer assays, among which only a fraction have been properly validated by prospective outcome studies or by measurement in plasma samples from outcome studies. ELISA and ELISA-derived assays have the highest sensitivity (above 95\%) and can be used to rule out VTE in patients with a low or intermediate clinical probability of DVT or PE, or, when using twolevel prediction rules for clinical assessment, in patients with an "unlikely" ruling for DVT or PE. In contrast, latex-derived assays have a lower sensitivity (around 90 to $95 \%$ ) and must be restricted to patients with a low clinical
\end{abstract}

probability or a DVT or PE "unlikely" ruling. No D-dimer assay is sensitive enough to allow safely ruling out VTE in patients with a high clinical probability, a population in whom D-dimer results are very rarely negative.

In outpatients, D-dimer measurement combined with clinical assessment should be the first step in a diagnostic algorithm for DVT or PE as it allows ruling out VTE in around $30 \%$ of patients with a clinical suspicion of VTE. In other populations such as inpatients, elderly patients, patients with cancer, or pregnant women, its use should be considered on a case-by-case basis due to a significantly lower diagnostic yield. Efforts are under way to simplify the decision rules for assessing clinical probability and establishing variable cut-offs for D-dimer to improve their exclusion rate in the elderly. 


\title{
Cerebral vein thrombosis
}

\author{
José Ferro
}

Department of Neurosciences, Hospital Santa Maria, Lisboa, Portugal

Dural sinus and cerebral vein thrombosis (CVT) are less common than other stroke types but more difficult to diagnose. Clinical presentation of CVT varies in different demographic groups (children, women, elderly) and is also influenced by the interval onset-diagnosis, the presence of parenchymal lesions, the site of the venous occlusion and the underlying disease. The current diagnostic standard for CVT consists of MR with T2* sequences and angio-MR. Venous CT angiography has probably the same diagnostic accuracy as veno-MR.

Multiple conditions, including contraceptives, infections and cancer predispose to CVT. Screening for genetic (protein $\mathrm{C}$ and $\mathrm{S}$, anti-thrombin III, factor V Leiden, prothrombin mutation) and acquired prothrombotic conditions (anti-phospholip syndrome) should be performed in all patients, even in those with another apparent cause. The prognosis of CVT can be stratified using the validated CVT risk score. Anticoagulation (IV heparin or preferably SC LMWH) remains the fundamental therapeutic measure in the acute phase. In comatose patients or in those who deteriorate despite anticoagulation, more aggressive interventions may be used: hemicraniectomy is life-saving if there is a brain lesion with severe mass effect, while local IV thrombolysis may be considered if there is no mass effect or the deep venous system is occluded. Antiepileptic drugs are recommended in patients with seizures and in those with supratentorial hemorrhagic lesions. Patients with CVT have a moderate risk of further thrombotic events, mainly extra-cerebral, but the length of anticoagulation ( 3 to 12 months or life-long) after CVT is uncertain and needs to be established by clinical trials. The efficacy and safety of aggressive interventions for the treatment of acute CVT will also be evaluated in planned trials and registries. 


\title{
Moderate hyperhomocysteinemia: marker or cause of a disease?
}

\author{
Rosanna Abbate, Rossella Marcucci, Gabriele Cerini, Giulia Degl'Innocenti
}

Dipartimento di Area Critica medico Chirurgica, University of Florence, Florence, Italy

Pathophysiological and clinical data suggest a casual role of moderate hyperhomocysteinemia for vascular disease, but inconclusive data are present on a reduction of cardiovascular events associated with the correction of hyperhomocysteinemia.

Several mechanisms, proposed by experimental studies, demonstrate that moderate hyperhomocysteinemia can determine a vascular injury by endothelial prothrombotic activation, oxidative stress and hypercoagulability.

It is well know that homocysteine circulating levels are dependent on B12, B6 and folic acid levels or genetic alteration of enzymes such as MTHFR (methylenetetrahydrofolate reductase) or CBS (cystathionine beta-synthase).

VISP, NORVIT and HOPE 2 trials were designed on the basis that vitamin supplementation with B-vitamins and folic acid could reduce vascular risk of about 30\%. On the other hand, a large meta-analysis of previous observational studies found that a $25 \%$ reduction in Hcy levels might determine a reduction of $11 \%$ in cardiovascular risk and
$19 \%$ in cerebrovascular risk.

Furthermore, these large perspective trials have enrolled patients with a previous vascular event. It is conceivable that reduction of Hcy in these patients could have an effect which is diluted by the concomitant antihypertensive, anticoagulant and hypolipidic therapies. An extended follow-up of these trials could have enhanced their statistical power, by increasing the number of vascular events.

Finally, even if these trials have enrolled a large number of patients, the sample size could be not enough. Indeed, it has been calculated - on the basis that a reduction of $25 \%$ in Hcy levels should determine a $10 \%$ reduction of vascular events - that a trial should enroll about 52000 patients to have enough statistical power.

Therefore, intervention studies available are not able to answer the question if moderate hyperhomocysteinemia is cause of vascular disease. They have demonstrated only that folic acid, vitamins B6 and B12 do not protect from clinical recurrences in patient with hyperhomocysteinemia. 


\title{
Pharmacoepidemiology of gastrointestinal bleeding complications of antiplatelet agents
}

\author{
Angel Lanas
}

Servicio de Aparato Digestivo, Hospital Clínico Universitario, University of Zaragoza. CIBERehd. ICS

Aspirin reduces the risk of cardiovascular events, but it is well documented that it can also damage the gastrointestinal (GI) tract. Overall, cardiovascular doses of aspirin increase the risk of upper GI bleeding two-fold. The damage can be extended beyond the upper gastrointestinal tract, but the clinical relevance of this remains to be determined. It is poorly understood why some patients have increased risk of complications whereas most people taking low-dose aspirin develop only minor, clinically irrelevant lesions. A number of risk factors can be used to determine which patients are more likely to develop aspirin-associated GI bleeding; these include a previous GI ulcer, ulcer complications, dyspepsia, Helicobacter pylori infection and concomitant drug therapy with non-steroidal anti- inflammatory drugs (NSAIDs), COX-2 selective inhibitors or clopidogrel. Aspirin-induced GI damage can be reduced and a number of strategies can be implemented to shift the risk-benefit ratio in favor of aspirin. Proton pump inhibitors are more effective than $\mathrm{H} 2$-receptor antagonists in preventing dyspeptic symptoms, peptic ulcers and bleeding ulcers in aspirin users. H. pylori infection is a risk factor of aspirin-induced ulcer bleeding, but the role of $\mathrm{H}$. pylori eradication in the prevention of this outcome requires further investigation. The individual assessment of the benefits and risks with aspirin, based on the underlying GI and cardiovascular risk factors, is the key to successful therapy. 


\title{
Antithrombotic agents after vascular surgery
}

\author{
David Bergqvist
}

Department of Surgical Sciences, Uppsala University Hospital, Uppsala, Sweden

\begin{abstract}
Antithrombotic therapy in connection with vascular surgery has several aims: to prevent graft/reconstruction failure, to maintain limb salvage, to increase survival and in case of carotid artery surgery to prevent cerebral embolization. Postreconstructive failure is difficult to study because the pathogenesis is multifactorial and the time course is complex. Several factors are of importance for reconstruction failure such as run-off situations, surgical/technical errors, graft material, smoking habits, the presence of diabetes, haemostatic defects, activation of the coagulation system in connection with surgery etc. Locally there are also several thrombogenic stimuli such as synthetic graft material, the anastomosis with suture material, denuded endothelium and remaining arteriosclerotic plaques. Heparin as an angicoagulant in connection with clamping the arterial flow during vascular reconstruction has been used since the 1940's. Its use is
\end{abstract}

based on experience and seems pathophysiologically logical, when the blood flow is stopped, although there are no RCT's to support its use versus no anticoagulant. This use of heparin is reflected in a recommendation in the ACCP guidelines. When analyzing the evidence to fulfil the above mentioned aims there are data from RCT that early graft failure and occlusion can be prevented by platelet inhibitors, dextran, heparins and vitamin $\mathrm{K}$ antagonists. Late graft patency can be increased by platelet inhibitors, vitamin $\mathrm{K}$ antagonists and smoking cessation. Survival is increased by vitamin $\mathrm{K}$ antagonists and antiplatlet agents. Cerebral embolization is prevented by dextran in the early phase and later on by antiplatelet agents. Although not pure antithrombotic substances, the role of statins will be mentioned. Finally, the question will be asked how the above mentioned results are reflected in guidelines such as ACCP and TASC II. 


\section{What is new on the clinical aspects of fibrinolysis and thrombolysis?}

José A. Páramo

Hematology Service, University of Navarra, Pamplona, Spain

Whereas reduced overall fibrinolytic potential can be considered as a risk factor for both venous and arterial thrombosis, studies on the individual components gave conflicting results, and little evidence exists for a role of plasminogen, $\alpha 2$-antiplasmin, tissue-type plasminogen activator (t-PA), plasminogen activator inhibitor (PAI-1) and thrombin activatable fibrinolysis inhibitor (TAFI). These inconsistent results could be a consequence for the nonfibrinolytic properties of the fibrinolytic proteins, including their active role in inflammation, vascular remodeling, atherosclerosis and metabolic syndrome. The plasminogen activation system is upregulated in chronic inflammatory states including atherosclerosis, arthritis and infection, a view that is further supported by recent data from transgenic mice. The binding of plasmin to a variety of cells, including monocytes and endothelial cells, suggests its participation in different clinical scenarios.

Regarding thrombolysis, rt-PA is a well established reperfusion agent during the acute phase of acute myocardial infarction and stroke.
Therapeutic strategies for stroke have been developed with two main aims: restoration of cerebral flow and the minimization of the deleterious effects of ischemia on neurons. Over the last two decades, intense research has witnessed significant therapeutic advances in the thrombolytic field, but until today only one FDA-approved drug is available for ischemic stroke; i.e. rt-PA, whose usage is limited by a short therapeutic window and a risk of intracranial haemorrhage. Sonothrombolysis appears to be a promising procedure for the noninvasive or minimally invasive stroke management. Some metalloproteases (MMPs) have direct proteolytic activity against fibrin. Preclinical studies from our group have shown that MMP10 , in combination with reduced rt-PA dose, has the potential to be a fast and effective thrombolytic without inducing a systemic lytic state in mice models of arterial thrombosis. Further trials are needed to identify those patients who are most likely to benefit from treatment and the environment in which thrombolysis may be best in routine practice. 


\title{
Thromboprophylaxis in 'minor' orthopedic surgery
}

\author{
Henri Bounameaux
}

Division of Angiology and Hemostasis, University Hospital of Geneva, Geneva, Switzerland

Strong (Grade 1A) recommendations have been issued by the American College of Chest Physicians (ACCP) evidence-based clinical practice guidelines with respect to thromboprophylaxis after total hip or knee replacement, and following hip fracture. Grade 1 recommendations imply that benefit clearly outweigh risk, while Grade 2 suggestions imply that the benefit-risk ratio is not clear. The suffixes A-C describe the methodological quality of the data supporting the recommendation/suggestion.

Recommendations concerning 'minor' orthopedic interventions or leg fractures/trauma are based on lower methodology studies and are definitely weaker. According to the 8th ACCP Consensus Conference (Geerts WH et al. Chest 2008; 133:381S-453S), patients undergoing knee arthroscopy should not use routine thromboprophylaxis other than early mobilization (Grade 2B). Should they have additional thromboembolic risk factors or should the procedure have been complicated, LMWH prophylaxis is recommended (Grade 1B). For patients with isolated lower-extremity injuries distal to the knee treated surgically or with plaster cast), the suggestion is not to use routine thromboprophylaxis (Grade 2A).

Additional risk factors include previous thromboembolic events, malignancy, obesity, use of oral contraceptives or postmenopausal hormone therapy, presence of varicose veins. Should the bleeding risk be considered high, mechanical prophylaxis could be an option though not always applicable in case of leg trauma or surgery. Pharmacologic thromboprophylaxis should be reconsidered as soon as the bleeding risk has diminished. Although the ACCP consensus conference was unable to provide recommendations, it seems reasonable, if thromboprophylaxis is to be used in 'minor' orthopedic surgery, to use it for 7-14 days or until full mobilization, on a case-by-case evaluation.

In addition, patients should be encouraged to ambulate early and frequently after the procedure, and they should be made aware of the symptoms of venous thromboembolism so that they will present for investigation if there is a reasonable suspicion of such a complication. 


\title{
Highlights of the 5th International Conference on Thrombosis and Hemostasis Issues in Cancer (ICTHIC) 2010
}

\author{
Anna Falanga
}

Division of Immunohematology and Transfusion Medicine \& Hemostasis and Thrombosis Center, Department Oncology/Hematology, Ospedali Riuniti, Bergamo, Italy

Cancer patients have an increased risk for venous thromboembolism (VTE). This risk varies widely between patients and over the natural history of malignancy. Recent data identify multiple clinical risk factors and biomarkers to predict VTE. Targeted thromboprophylaxis utilizing model-based approaches may provide an optimal riskbenefit ratio, and are currently being explored.

Among biomarkers, much interest is arising from the study of microparticles (MP): these submicrometric membrane fragments circulate in plasma, and bring on their surface an ample variety of molecules, including tissue factor (TF) and procoagulant phospholipids. The MP-associated procoagulant factors allow the initiation and propagation of thrombotic phenomena within the blood vessels. Variations in MP levels and phenotypes make them relevant pathogenic markers of thrombotic disorders. Some studies show that tumors per se produce and release their own TF-bearing MP, and that high levels of these MP are associated to a higher risk of VTE in cancer patients. Much interest is focused on the management of thrombosis in cancer patients. The latest guidelines from both European and American Scientific Societies are matters of extensive discussion. It clearly appears that while there is a wide consensus for recommending primary thromboprophylaxis in acutely ill hospitalized cancer patients, differently, the use of thromboprophylaxis in patients undergoing ambulatory anti-cancer therapies is still controversial. In this case prophylactic measures to prevent VTE are not yet routinely recommended, with the exception of multiple myeloma patients treated with thalidomide (or analogs) plus steroids or chemotherapy. There is increasing evidence for a role of prolonged prophylaxis in high-risk surgical patients and in selected patients bearing central venous devices. Regarding treatment of symptomatic VTE, all guidelines agree that, for the long-term treatment, lowmolecular-weight heparins are to be preferred to vitamin $\mathrm{K}$ antagonists. However, the role of the new anticoagulants in the setting of VTE management in cancer is of interest and is starting to be explored. 


\title{
A renaissance of the contact phase of blood coagulation
}

\author{
Uri Seligsohn
}

Amalia Biron Research Institute of Thrombosis and Hemostasis, Sheba Medical Center, Israel

Until 1991, it was maintained that the intrinsic coagulation system is initiated by exposure of blood to negatively charged surfaces triggering activation of factor (F) XII in the presence of prekallikrein (PK) and high-molecularweight kininogen (HK). FXIIa then activates FXI, and FXIa activates FIX leading through additional reactions to thrombin generation. This sequence was difficult to accept because FXII, PK and HK deficient patients have no bleeding tendency, whereas FXI deficient patients do have injury-related bleeding. In 1991, this issue was resolved; thrombin generated by the tissue factor pathway was shown to activate FXI thereby bypassing contact activation. Consequently, the role of the contact phase of clotting became dubious. Other work indicated that FXIa promotes thrombin generation also after clot formation, and that thrombin activates thrombin activatable fibrinolysis inhibitor which removes lysine residues from fibrin, the anchors of plasminogen and tissue plasminogen activator. Notably, patients with severe FXI deficiency particularly bleed following surgery at site where there is increased fibrinolytic activity. Thus, FXI has a dual role: a procoagulant and an antifibrinolytic component. More recently, FXI deficiency in animals was shown to be protective against arterial and venous thrombosis without compromising hemostasis. Similarly, patients with severe FXI deficiency were found to be protected against ischemic stroke and deep vein thrombosis but not against myocardial infarction. Interestingly, severe FXII deficiency in mice was also protective against ischemic stroke suggesting that FXII is important in thrombosis yet unimportant for Hemostasis. Because patients with severe FXI deficiency rarely bleed spontaneously and deficient animals do not display bleeding, means to induce FXI deficiency are sought for development of drugs aimed at prevention and treatment of thrombosis. 


\title{
News on the anticoagulant pathway of blood coagulation
}

\author{
Javier Corral, Irene Martínez-Martínez, Vicente Vicente
}

University of Murcia. Centro Regional de Hemodonación, Murcia, Spain

\begin{abstract}
Three main anticoagulants with differential setting and targets keep blood flowing under normal conditions, regulate blood clot propagation and ensure that the clotting process remains as a local process.

The tissue factor pathway inhibitor regulates the initial steps of blood coagulation involving FVIIa and TF, and consequently inhibits newly formed FXa associated with the TF-FVIIa complex. Recently, FVIIa inhibition by antithrombin and EPCR has also been described.

The other two existing anticoagulants are functionally associated with thrombin. The activated protein $\mathrm{C}$ (aPC), generated by thrombin bound to thrombomodulin, modulates blood coagulation by cleaving a limited number of peptide bonds in factor VIIIa (FVIIIa) and factor $\mathrm{Va}(\mathrm{FVa})$, cofactors in the activation of factor $\mathrm{X}$ and prothrombin. In contrast, thrombin and other procoagulant serine proteases are targets of antithrombin, a serpin with an efficient inhibitory mechanism that is activated by endothelial glycosaminoglycans or heparin.
\end{abstract}

A number of genetic defects, including common polymorphisms, affecting the aPC pathway ( $\mathrm{PC}$, its cofactor PS, and FV) and antithrombin constitute risk factors of venous thrombosis. The search of genetic defects has allowed the identification of new mechanisms, including changes in glycosylation or conformational consequences in the protein, involved in the deficiency of these anticoagulants. Moreover, these studies may help to identify deficiencies with increased risk of thrombosis by a gain of function supplementary to the loss of function. The effect of different environmental factors or drugs that might cause acquired deficiency of antithrombin and might contribute to explain the risk of thrombosis identified in different settings has also been evaluated.

It is important to point out other functions of these anticoagulants, particularly the anti-inflammatory potential of aPC and antithrombin as well as their potential therapeutic use and controversies.

Finally, there are new data sustaining a role in arterial thrombosis for these anticoagulants. 